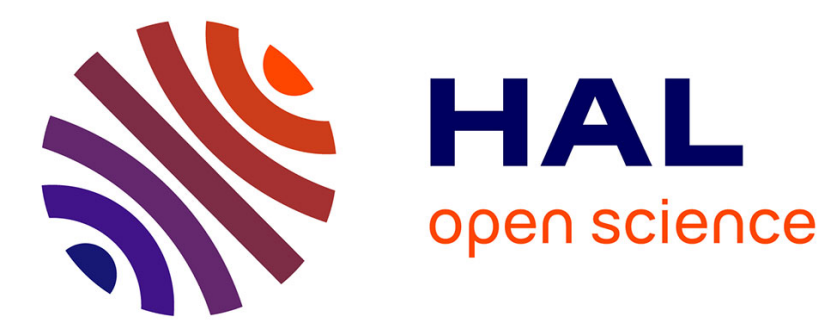

\title{
N-Conductor Passive Circuit Modeling for Power Converter Current Prediction and EMI aspect
}

Roberto Mrad, Florent Morel, Gaël Pillonnet, Christian Vollaire, Philippe Lombard, Angelo Nagari

\section{To cite this version:}

Roberto Mrad, Florent Morel, Gaël Pillonnet, Christian Vollaire, Philippe Lombard, et al.. N-Conductor Passive Circuit Modeling for Power Converter Current Prediction and EMI aspect. IEEE Transactions on Electromagnetic Compatibility, 2013, 55 (6), pp.1169-1177. 10.1109/TEMC.2013.2265048 . hal-01103586

\section{HAL Id: hal-01103586 \\ https://hal.science/hal-01103586}

Submitted on 15 Jan 2015

HAL is a multi-disciplinary open access archive for the deposit and dissemination of scientific research documents, whether they are published or not. The documents may come from teaching and research institutions in France or abroad, or from public or private research centers.
L'archive ouverte pluridisciplinaire HAL, est destinée au dépôt et à la diffusion de documents scientifiques de niveau recherche, publiés ou non, émanant des établissements d'enseignement et de recherche français ou étrangers, des laboratoires publics ou privés. 


\title{
N-Conductor Passive Circuit Modeling for Power Converter Current Prediction and EMI aspect
}

\author{
Roberto Mrad, Florent Morel, Member, IEEE, Gael Pillonnet, Member, IEEE, Christian Vollaire, Member, IEEE, \\ Philippe Lombard, and Angelo Nagari, Member, IEEE,
}

\begin{abstract}
This paper proposes a method to study the impact of passive circuits such as filters, loads, and board layout on conducted electromagnetic emissions. The method is dedicated to power converters with $\mathbf{N}$ active conductors and a ground conductor, followed by passive circuits. A sophisticated use of impedance matrices allows high frequency current prediction. The power system is divided into functional circuits called blocks. Each passive block is modeled by an impedance matrix. A fast matrix calculation permits association of those matrices to obtain a compact model represented by an impedance matrix. Knowing the converter output voltages and the resulting impedance matrix, currents are calculated to study the influence of each passive block on high frequency current spectrum, and so the EMI (electromagnetic interference). This will help for filter and board layout design for a given load. After presenting the general use of the method, the validation is performed on a differential Class-D audio amplifier used in cell phone applications. The practical application on a two-active conductor system validates the method up to $110 \mathrm{MHz}$.
\end{abstract}

Index Terms-Conducted EMI, Passive circuit modeling, Impedance matrix, current prediction, Class-D amplifier.

\section{INTRODUCTION}

Today's electronic applications are mostly dedicated to embedded systems, where autonomy is a major issue. Thus, power management circuits are widely used to increase power efficiency and extend battery life. Nevertheless, those are switching circuits producing high levels of electromagnetic (EM) disturbances, in particular when delivering high supply currents. Moreover, many power converters are often used at a time to fulfill all the power requirements, needed for the multitude of applications in a single device. To increase their electro-magnetic compatibility (EMC), engineers rely on many control methods, such as spread spectrum [1]-[4], slew rate control [5], [6], pulse position [7], noise shaping [8] and dead time compensation [9]. Nevertheless, in most cases these methods are not enough and use of electro-magnetic interference (EMI) filters is unavoidable.

Class-D audio amplifiers are an example of power converters in embedded systems. This type of converter is used in cell phones because of its high efficiency. Moreover, the design of today's Class-D amplifiers provides high audio quality. However, it delivers high power to the speaker (up to $2.3 \mathrm{~W}$ [1]) through switching voltages/currents (around $500 \mathrm{kHz}$ with less $10 \mathrm{~ns}$ rise/fall time). With switching, high frequency harmonic, ringing and overshoot, this amplifier is a major source of EM disturbance in cell phones. For this reason, an EMI filter is usually inserted at the output to prevent disturbance through the PCB. Nevertheless, such passive circuits are very bulky and occupy relatively a huge area in a cell phone compared to the amplifier size. For that, a well designed filter is much needed to reduce the cost and filter area.

The choice of EMI filters is generally based on expertise and their performance is tested on prototypes. For that, EMI methods and models are greatly needed before production. However, traditional methods such as time domain simulations become exponentially complicated when they are used for EMI issues. Therefore, designers use frequency models, called also macro-models. Macro-models are frequently used for EMI purposes because quite often system integrators have little information about the components used and their studies are based on measurements. Such models have good accuracy, but in most cases they are dedicated to a specific application or specific problem so their use cannot be generalized. In previous work, methods like [10]-[12] study only the Common Mode (CM) and their accuracy does not exceed $30 \mathrm{MHz}$. In [13]-[15], the method has two different models for common and Differential Modes (DM), but it is not able to take into account the mode conversion. Methods in [16]-[18] study the EMI performance of a single part from the system, but do not deal with system EMI performance and EMI emissions. Otherwise, [19] takes into account the system emissions, but the model is very compact and cannot be used for system improvement.

In this paper, a frequency model for converter systems with $\mathrm{N}$ active conductors and ground reference is presented. The major goal is to improve system design in terms of EMC. Using segmentation, a well-known technique, the present approach allows the designers to separate the different system parts into blocks and detect the EMI defects. Then, a library of blocks can help designers improve their design at the early stages. In addition, common mode, differential model and mode conversion are taken into account. Finally, this approach generates a compact macro-model which can be used in further EMI simulation. Having a short simulation time, this model can be easily used in an optimization process.

After segmentation, passive blocks are modeled by impedance matrices. These are an accurate frequency model for passive and linear circuits. They can take into account the system non-idealities, such as stray elements and impedance of the PCB track in a simple way, without having to search for all the stray elements in order to have the correct high frequency behavior. Moreover, many simulation programs offer the possibility to import network matrices (usually as S-parameters), such as ADS Agilent, Saber and CST, etc. However, Nport impedance matrix theory is not clearly explained in the 


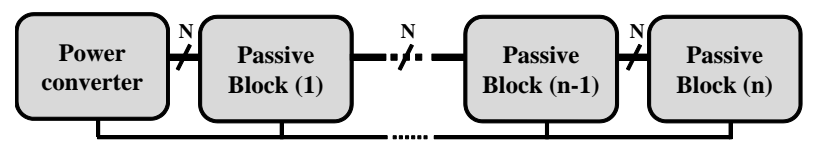

Fig. 1. System decomposition into blocks.

literature. Thus, this paper develops the required theory as well as the calculation details. In addition, three methods for impedance matrix determination are presented, and then the validity and the limitation of each one are discussed.

Section II explains the theoretical background for the method, describing the case of a converter with $\mathrm{N}$-conductors. Section III discusses the details for the method application on a Class-D audio amplifier system with 2 active conductors. Finally, the conclusion in section IV.

\section{PASSIVE CIRCUIT MODELING AND CURRENT}

\section{PREDICTION FOR N-ACTIVE CONDUCTOR CIRCUITS}

In the proposed method, the considered power system is divided into functional sub-circuits which are called blocks. The system is represented by a chain of blocks as shown in Fig. 1. In this chain, on the one hand there are the active blocks, such as switching amplifiers and power converters etc. On the other hand, there are the passive blocks such as filter, load and tracks, etc. Note that passive blocks can also be divided into several blocks if needed. The sub-blocks are modeled in the same way as a normal block. This will help improve the design of a given system part, such as filter and PCB layout. However, the following requirements must be verified before applying this method:

- No feedback control for the converter coming from the following passive blocks. In the opposite case, output voltages are dependent on the passive circuit which makes the model invalid.

- No significant inductive, capacitive or electromagnetic coupling between blocks. In other words, a block impedance matrix is not related to other blocks which means their is no block behavior change after segmentation.

- Block should be sufficiently short to be considered electrically short (block dimensions $\ll$ wavelength).

- Passive components are used in their linear region (no saturation effects and low or no hysteresis effect, etc.).

\section{A. Active blocks model}

Active blocks with $\mathrm{N}$ active conductor can be modeled with $\mathrm{N}$ electric sources and a $2 N \times 2 N$ impedance matrix as shown in Fig. 2. Determining the electric sources and the output impedance matrix cannot be generalized. Each particular circuit has its dedicated set of measurements. Many measurement possibilities can be imagined, such as: open circuit (if possible), loaded, open circuit when shorting (if possible) or loading other ports, etc. Some, examples for output impedance extraction can be found in [20]-[24]. However, in some cases, depending on the converter output impedance, the filter impedance and the considered frequency range, the

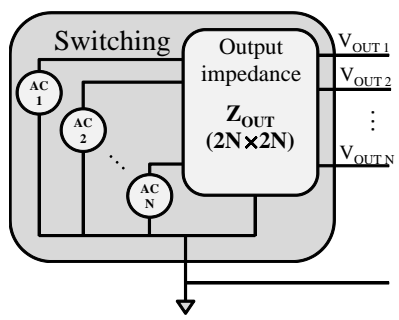

Fig. 2. Active block model.

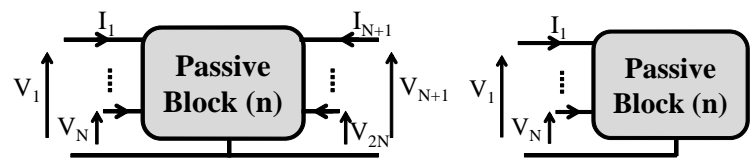

Fig. 3. Type A : $2 N+1$ ports.

Fig. 4. Type $B: N+1$ ports.

output impedance matrix can be neglected. In this case, the converter will be modeled only by $\mathrm{N}$ electric sources that model the converter switching.

\section{B. Passive blocks types and models}

Passive blocks are modeled by impedance matrices relating voltages to currents. Two types of blocks can be distinguished, for simplification, they will be called Type $A$ and Type B blocks for the rest of this paper. Type $A$ is intended for the blocks situated in the middle of the chain and can be connected from both sides with $2 N+1$ ports ( $\mathrm{N}$ inputs and $\mathrm{N}$ outputs plus a ground) as shown in Fig. 3. Type $B$ is intended for blocks situated at the end of the chain and can be connected only from its inputs with $N+1$ ports (Fig. 4). More precisely, Type $B$ blocks are intended for loads because usually they only have an electrical input but with other types of physical output (mechanical, thermal and acoustic, etc.). According to Fig. 3, Type A blocks have $2 N$ voltages and $2 N$ currents, so their impedance matrix dimension is $2 N \times 2 N$ as shown in equation (1).

$$
\left(\begin{array}{c}
V_{1} \\
\vdots \\
V_{2 N}
\end{array}\right)=\left(\begin{array}{ccc}
z_{11} & \ldots & z_{12 N} \\
\vdots & \ddots & \vdots \\
z_{2 N 1} & \ldots & z_{2 N 2 N}
\end{array}\right) \cdot\left(\begin{array}{c}
I_{1} \\
\vdots \\
I_{2 N}
\end{array}\right)
$$

Similar to Type $A$ blocks, Type $B$ blocks have $N \times N$ impedance matrix because they have $N$ voltages and $N$ currents as shown in equation (2).

$$
\left(\begin{array}{c}
V_{1} \\
\vdots \\
V_{N}
\end{array}\right)=\left(\begin{array}{ccc}
z_{11} & \ldots & z_{1 N} \\
\vdots & \ddots & \vdots \\
z_{N 1} & \ldots & z_{N N}
\end{array}\right) \cdot\left(\begin{array}{c}
I_{1} \\
\vdots \\
I_{N}
\end{array}\right)
$$

\section{Impedance matrix determination}

Impedance matrix determination can be done using several methods and in this paper three methods are studied. The first uses measurements with an Impedance Analyzer (IA), the second uses measurements with a Vector Network Analyzer 


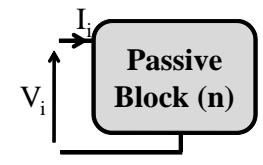

Fig. 5. $Z_{i i}$ measurements.

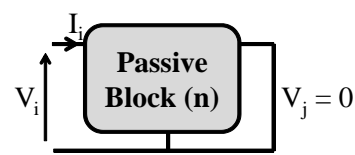

Fig. 6. $Z_{i j_{s c}}$ measurements.
(VNA) and the third uses simulation with Advanced Design System (ADS) program [25]. Note that the impedance matrix for passive circuits is symmetric with respect to the diagonal due to the reciprocity theorem $\left(Z_{i j}=Z_{j i} ; \forall i ; \forall j ; i \neq j\right)$.

1) Impedance matrix determination by IA: Determining an impedance matrix using an IA requires 2 steps. First of all, diagonal elements are measured. Equation (3) and Fig. 5 show that $z_{i i}$ is the input impedance of the $i$ port.

$$
z_{i i}=\left.\frac{V_{i}}{I_{i}}\right|_{I_{j}=0} \quad ; \quad i \neq j \quad ; \quad j=1 \ldots(2) N
$$

In practical term, the IA is connected directly to port the $i^{t h}$ port to measure $z_{i i}$ when the remaining ports are open circuits $\left(I_{j}=0 A ; j \neq i\right)$.

The second step is to measure the off-diagonal elements $\left(z_{i j} ; i \neq j\right)$. Equation (4) shows how to determine the cross elements theoretically.

$$
z_{i j}=\left.\frac{V_{i}}{I_{j}}\right|_{I_{i}=0} \quad ; \quad i \neq j \quad ; \quad i=1 \ldots(2) N
$$

Nevertheless, it is not possible to apply this in practice with a single port IA and it is delicate to apply with dual-port IA. According to equation (4), this method needs a current injection on the $j^{t h}$ port and exact voltage amplitude and phase sensing on the $i^{t h}$ port with $I_{i}=0 \mathrm{~A}$. Instead, equation (5) gives an alternate user-friendly method for the determination of the cross elements.

$$
z_{i j}=\sqrt{z_{j j}\left(z_{i i}-z_{i j_{s c}}\right)} \quad ; \quad i \neq j
$$

Where $z_{i j_{s c}}$ is the impedance measured from the $i^{t h}$ port when the $j^{\text {th }}$ port is in Short Circuit (SC) (Fig. 6), $z_{i i}$ is the $i^{t h}$ diagonal element presented by (3), $z_{i j}$ is the $i^{t h} j^{t h}$ element presented in (4). However, if the difference between $z_{i i}$ and $z_{i j_{s c}}$ is smaller than the minimum accuracy of the IA, equation (5) may cause some errors.

2) Impedance matrix determination by VNA: A VNA can measure the scattering matrix. To determine an impedance matrix of a passive block, the scattering matrix is measured, so (6) can be used to convert it to an impedance matrix.

$$
Z=(I-S)^{-1} \cdot(I+S) \cdot Z_{0}
$$

$Z$ is the impedance matrix, $S$ is the scattering matrix, $I$ is an identity matrix and $Z_{0}$ is the characteristic impedance of the VNA (usually $50 \Omega$ ).

Note that, a VNA can introduce significant measurement uncertainty. The latter depends on the frequency range, the element type (diagonal or cross element) and the measured impedance level compared to $50 \Omega$ [26]-[29].

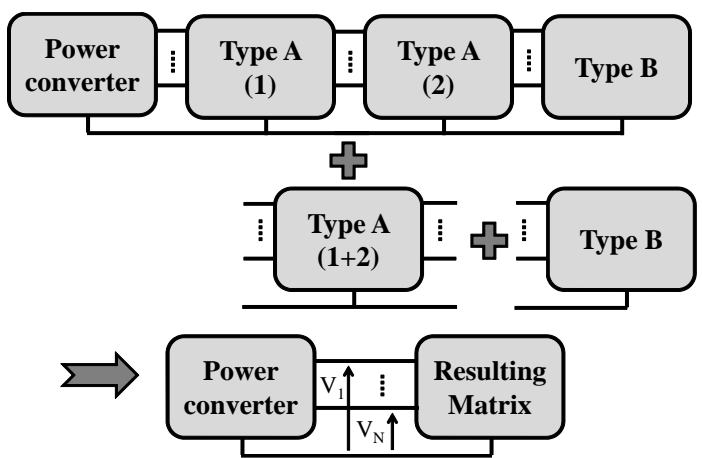

Fig. 7. Block association technique.

3) Impedance matrix determination by simulation: The advantage of simulation is determining the impedance matrices before construction, so the designer needs to build fewer prototypes. Moreover, the only frequency band limitation is the modeling details, which means the model validity. Otherwise, the band can be chosen by the user. Also, if needed, it is possible to study the influence of the uncertainty and the disparity of the components due to the fabrication procedure [30].

\section{Impedance matrix association}

Impedance matrices can be associated in order to get one resulting matrix, containing their impedance behavior seen by the converter as shown in Fig. 7. The latter also shows that there are two types of association. On the one hand, there is the Type $A+$ Type $A$ association, and on the other hand, there is the Type $A+$ Type $B$ association.

1) association of 2 Type A blocks: Two Type A blocks are considered to describe this association: The first called $k$ with an impedance matrix $Z_{k}$ and the second called $m$ with an impedance matrix $Z_{m}$. $Z_{k}$ and $Z_{m}$ are divided into 4 submatrices equal in terms of dimension $(N \times N)(7)$.

$$
Z_{k}=\left(\begin{array}{c|c}
Z_{k 11} & Z_{k 12} \\
\hline Z_{k 21} & Z_{k 22}
\end{array}\right) ; Z_{m}=\left(\begin{array}{c|c}
Z_{m 11} & Z_{m 12} \\
\hline Z_{m 21} & Z_{m 22}
\end{array}\right)
$$

Then, equation (8) gives the association method.

$$
Z_{k m}=\left(\begin{array}{cc}
Z_{k m 11} & Z_{k m 12} \\
Z_{k m 21} & Z_{k m 22}
\end{array}\right)
$$

with :

$Z_{k m 11}=Z_{k 11}-Z_{k 12}\left(Z_{k 22}+Z_{m 11}\right)^{-1} Z_{k 21}$

$Z_{k m 12}=Z_{k 12}\left(Z_{k 22}+Z_{m 11}\right)^{-1} Z_{m 12}$

$Z_{k m 21}=Z_{m 21}\left(Z_{k 22}+Z_{m 11}\right)^{-1} Z_{k 21}$

$Z_{k m 22}=Z_{m 22}-Z_{m 21}\left(Z_{k 22}+Z_{m 11}\right)^{-1} Z_{m 12}$

It is also possible to use the transfer matrix (matrix that gives the output voltages and currents in terms of the input voltages and currents [31]) for this association.

This type of association is not used in section III but has been validated in [32]. 
2) Type A and Type B blocks association: To explain how to associate a Type $A$ and a Type $B$ blocks the $\mathrm{km}$ block (Type $A \Rightarrow 2 N \times 2 N$ matrix dimension) presented before and a load block $Z_{L}$ (Type $B \Rightarrow N \times N$ matrix dimension) are considered. If $Z_{k m L}$ is the resulting impedance matrix, it can be calculated with equation (9). $Z_{k m L}$ is a $N \times N$ impedance matrix.

$$
Z_{k m L}=Z_{k m 11}-Z_{k m 12}\left(Z_{L}+Z_{k m 22}\right)^{-1} Z_{k m 21}
$$

In the resulting matrix $Z_{k m L}$, the diagonal elements $\left(Z_{i i} ; i=1 \ldots N\right)$ represent the input impedance of the passive blocks seen by the converter. The cross elements $\left(Z_{i j} ; i \neq j ; i=1 \ldots N ; j=1 \ldots N\right)$ represent the couplings between the active conductor through the passive system.

Note that the association techniques of two blocks Type A+Type A or Type A+Type B have been experimentally tested and validated in the case of simulations and measurements but the results will not be shown in next sections. [32] shows the experimental validation in the case of measurements.

\section{E. Current prediction}

After associating all the matrices of the passive blocks, one single impedance matrix is obtained called $Z_{R}(N \times N)$. This matrix is a compact model replacing all the passive blocks in terms of impedance seen by the converter. Knowing the output voltages spectra of the converter, the currents spectra can be calculated using equation (10).

$$
\left(\begin{array}{c}
I_{1} \\
\vdots \\
I_{N}
\end{array}\right)=\left(Z_{R}\right)^{-1}\left(\begin{array}{c}
V_{1} \\
\vdots \\
V_{N}
\end{array}\right)
$$

To have a real predictive method, voltages should be known (measured or simulated) without pre-specifying the load. To this aim, it is important that the input voltages are independent from the passive circuits behind. If the voltages are dependent to the load, the output voltages should be measured or simulated with the final passive blocks. In this case, the method is able to produce a compact model of the converter but it is not possible to predict the current spectrum.

\section{EXPERIMENTAL APPLICATION ON A TWO ACTIVE CONDUCTOR CLASS-D AMPLIFIER}

The method presented in section II was applied on a Class-D audio amplifier used in hands-free cell phone applications [33]. This amplifier is a switching Pulse Width Modulation (PWM) converter using the audio signal as reference (the modulation technique is shown in Fig. 8). In hands-free applications, the Class-D amplifier uses an H-Bridge power stage which gives a differential output ( 2 active conductors). In real applications, this amplifier is followed by an EMI filter, then a speaker.

The studied system has 2 active conductors, which means that the filter has a $4 \times 4$ impedance matrix and the load (speaker) has a $2 \times 2$ impedance matrix. The Class-D amplifier
TABLE I

FILTER COMPONENTS VALUES.

\begin{tabular}{|c|c|}
\hline$L_{1}, L_{2}$ & $15 \mu \mathrm{H}$ \\
\hline$C_{1}, C_{2}$ & $0.033 \mu \mathrm{F}$ \\
\hline$C_{4}, C_{5}$ & $0.068 \mu \mathrm{F}$ \\
\hline$C_{3}$ & $0.15 \mu \mathrm{F}$ \\
\hline$R_{1}, R_{2}$ & $22 \Omega$ \\
\hline
\end{tabular}

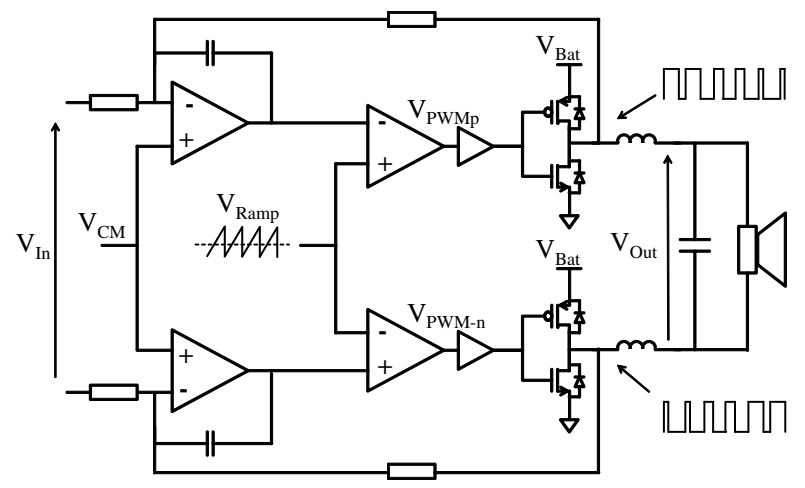

Fig. 8. PWM modulation for Class-D amplifier.

used in [34] is a test chip mounted on a test board. The filter (Fig. 9), used in [35] is made on a specific filter board for easy current sensing. The filter component values are given in TABLE I. Two different loads were studied; the first is a speaker used in cell phone applications [36], and the second is a dummy load (Fig. 10) that emulates the real speaker (note that the dummy load is considered for the rest of this paper). The dummy load component values are given in TABLE II. The different blocks were equipped with SMB connectors for easy association (disassociation) by a simple plug (unplug). The boards are shown in Fig. 11.

\section{A. Filter and load impedance matrix determination}

To determine the impedance matrix of the two blocks (filter and load) the 3 methods described previously are applied. The filter and load impedance matrices are determined with the same methods but in the following sections only the case of the filter is detailed for conciseness.

1) Filter impedance matrix determination using an IA: The IA is used following the procedure presented in section II-C1 to measure $Z_{i i}(i=1 \ldots 4)$ and $Z_{i j_{s c}}$ ( $i=1 \ldots 4 ; j=1 \ldots 4 ; i \neq j)$. Hereafter, equation (5) is used to calculate $Z_{i j}$. Note that the IA used has a frequency limitation of $110 \mathrm{M} \mathrm{Hz}$ so the measurements were done in the range of $[10 \mathrm{kHz}-110 \mathrm{MHz}$.

2) Filter impedance matrix determination using a VNA: The 4-port VNA is used to measure the filter scattering matrix. Then, equation (6) transforms the measured $4 \times 4$ scattering matrix to a $4 \times 4$ impedance matrix. In this case, $I$ is a $4 \times 4$ eye matrix and $Z_{0}$ is $50 \Omega$. Note that the minimum frequency possible for the VNA used is $300 \mathrm{kHz}$, so that the measurements are done in the frequency range [300 kHz - $1 \mathrm{GHz}]$.

3) Filter impedance matrix determination by simulation: In the simulation program used, PCB tracks are modeled 


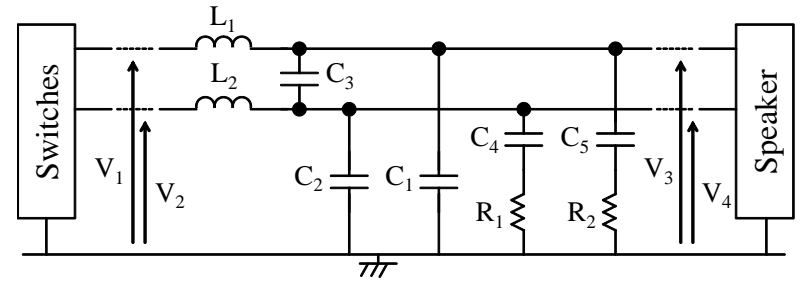

Fig. 9. The filter schematic used.

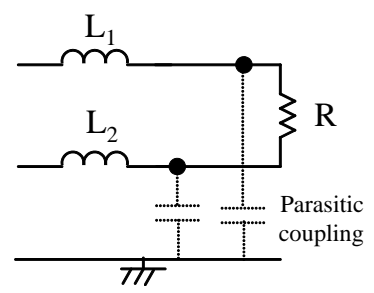

Fig. 10. The dummy load schematic.

as strip-lines. PCB material characteristics are taken into account. Components are replaced by the measured component impedance itself or by a specific model provided by the constructor. Filter layout is taken into account and in some cases where the tracks are close to each other, coupling between them is also added using specified strip-line blocks. However, the coupling between components themselves and between components and tracks are not modeled but it might be possible to take them into account [37]. This is the real advantage of the measuring methods shown before (IA and VNA) where these types of couplings are taken into account without any additional effort. The frequency band can be defined by the user and was defined as $[10 \mathrm{kHz}-1 \mathrm{GHz}]$.

4) Result comparison: To compare the results from the above three methods, the impedance results of $z_{11}$ (diagonal elements) are plotted in Fig. 12 and $z_{14}$ (cross elements) in Fig. 13. According to Fig. 12, all three methods give similar results for the diagonal elements. However, Fig. 13 shows that in the case of measurements using IA (grey line) or VNA (black line) there may be an accuracy problem with the cross elements caused by the precision of the measuring device.

In fact, in the case of IA, cross elements are calculated using equation (5), and $z_{11}$ is subtracted from $z_{14_{s c}}$. Nevertheless, at high frequencies $z_{11}$ is mainly equal to the filter inductor impedance and PCB stray capacitance; the capacitors impedances are very small. Moreover, at high frequencies $z_{14_{s c}}$ is approximately equal to $Z_{L_{1}}$ and the PCB stray capacitance ; the capacitor impedances are very small and $C_{2}$, $C_{4}, R_{1}$ are in short circuit (see Fig. 14). Hence, in Fig. 15, $z_{11}$ and $z_{14_{s c}}$ overlap at high frequencies which makes the difference less than the minimal precision of the measuring device (IA).

Otherwise, in the case of VNA, the $Z$ matrix is calculated from the filter $S$ matrix using equation (6). Fig. 16 shows the measured and simulated $s_{14}$. This figure shows that $s_{14}$ gets lower then the minimal measuring precision of the VNA in the [10 $\mathrm{MHz}-100 \mathrm{MHz}$ ] frequency band. This measurement

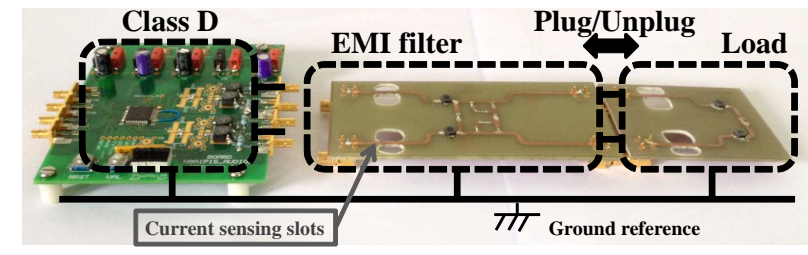

Fig. 11. Class-D amplification system.

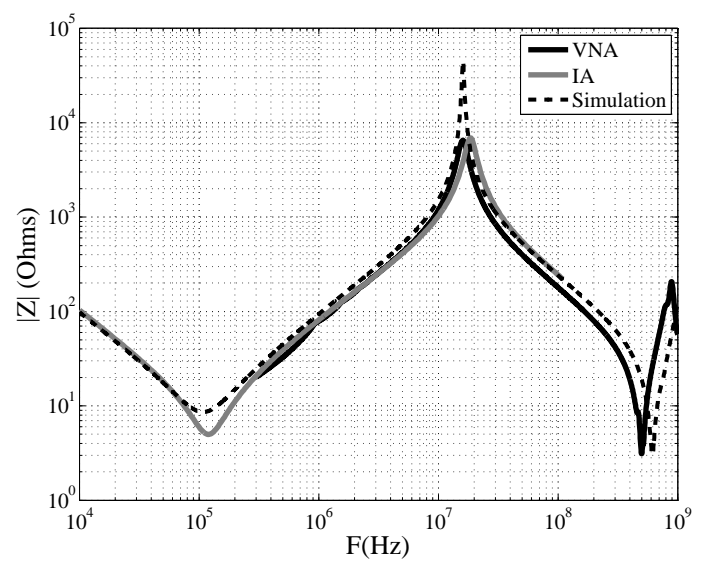

Fig. 12. Filter : $z_{11}$ impedance results.

error propagates to $Z_{14}$ which can be seen in Fig. 13 .

When using simulation, the impedances show mostly an over estimated resonance effects. In addition, it is not able to take into account all the inductive and capacitive coupling between tracks and components. However, it shows correct impedance behavior for the entire impedance matrix all along the considered frequency range. Moreover, determining an impedance matrix with simulation requires no prototype which means virtual EMI tests can be performed before production. Therefore, it is considered a good solution for this application, and these results are considered for the rest of this paper.

\section{B. Current prediction for Class-D amplifier}

First of all, the output voltages of the Class-D amplifier are measured with different loads (open circuit, speaker or filter and speaker). The frequency spectrum of these voltages is calculated using the Fast Fourier Transform (FFT) and the results are plotted in Fig. 17. According to Fig. 17 it is possible to say that the output voltages of the Class-D amplifier are quite independent of the load up to $110 \mathrm{MHz}$. This is due to closed loop voltage feedback, as the modulation is independent of the load, and also to the fact that the Class-D output impedance is negligible when compared to the load impedance. For that, the present method can be used for current prediction and the converter output matrix shown in Fig. 2 can be neglected.

The association method shown in section II-D2 is used in order to join both filter and load matrix in a compact model. This model is represented by a $2 \times 2$ impedance matrix and contains the impedance of all the blocks seen by the converter. The resulting matrix can be used for current prediction. 


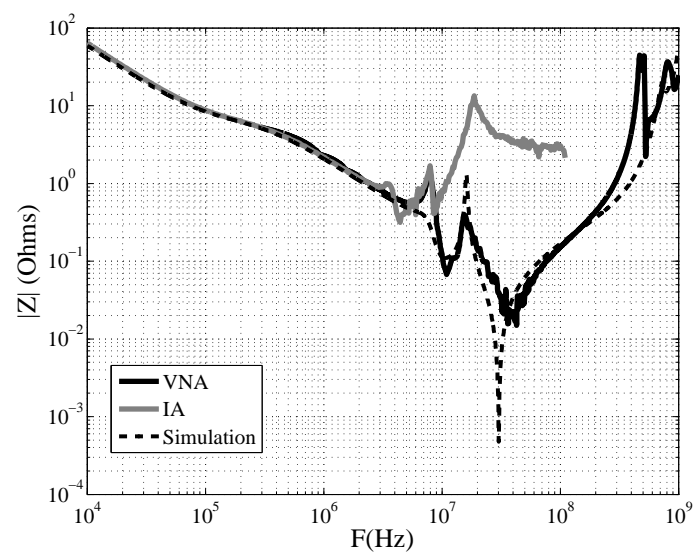

Fig. 13. Filter : $z_{14}$ impedance results.

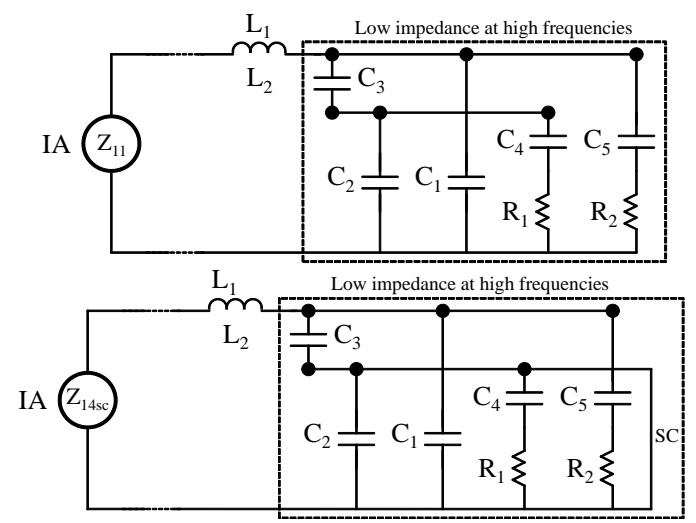

Fig. 14. $z_{11}$ and $z_{14_{s c}}$ impedances seen by the IA

A $10 \mathrm{kHz}$ sinusoidal signal is applied on the audio input of the Class-D amplifier. The power stage is supplied by $3.6 V$ (similar to the cell phone battery voltage). The output voltages for both active conductors were measured in the time domain at the same time. A 12-bit vertical quantification oscilloscope [38] is used in order to reduce the noise floor. Note that measuring the voltages at the same time makes the relative phase between the outputs correct, and can be used after FFT. Hence, knowing the output voltages and the resulting impedance matrix, equation (10) is used for current calculation. Results can be seen in Fig. 18 and only one of the two currents is shown because they are similar. As can be seen from this figure the calculated current spectrum is in good agreement with the measured one. The current is measured using a current probe with a bandwidth of $120 \mathrm{MHz}$ [39].

\section{CONCLUSION}

A new use of impedance matrices for EMI purposes was presented in this paper. The passive functional circuits of the system are modeled separately with impedance matrices. Those matrices are then associated to rebuild the system in a single one. The resulting matrix and converter output voltages (input voltages for the passive blocks) can be used for current calculation. If the output voltages of the converter are independent of the load, the open circuit output voltages can

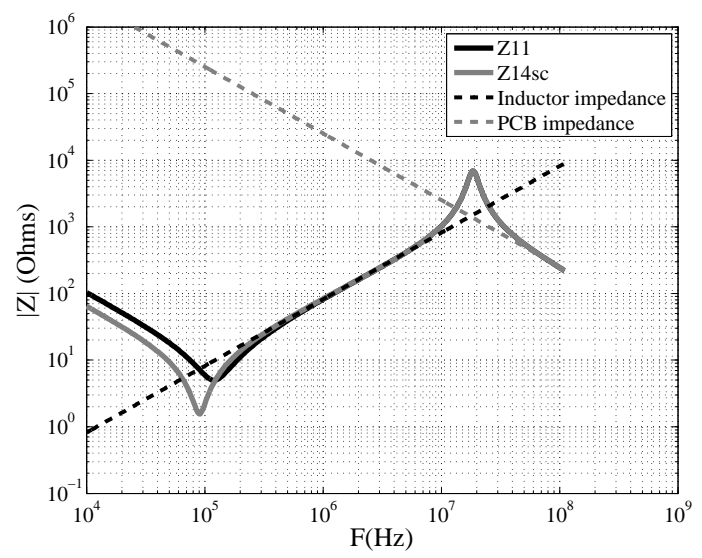

Fig. 15. Filter: Measured $z_{11}$ and $z_{14_{s c}}$. with IA

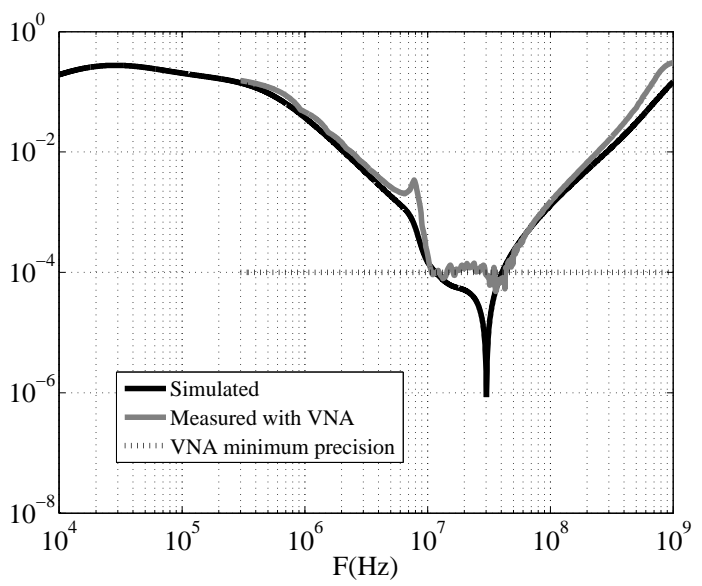

Fig. 16. Filter : VNA measuring precision seen in $s_{14}$.

be used for current prediction. This approach was applied to a cell phone audio system containing a Class-D audio amplifier. The impedance matrices are determined using three different methods. The theory and validity of each is discussed. Finally, the Class-D amplifier voltages and the resulting matrix for both the filter and the load were used for current calculation. Using the simulated filter and load impedance matrices good results were seen up to $110 \mathrm{MHz}$. The accuracy of these methods is due to the fact that the $\mathrm{CM}, \mathrm{DM}$ and mode conversion are included by default in the modeling process.

This method can be used by system integrators when using the ready-made integrated systems in order to search for the best filter design. The present method permits virtual measurements to be made in order to validate a filter or a routing design in terms of EMI performance, before board prototype construction. It is also possible to make a library of matrices corresponding to different filters and loads in order to check the use of an old design in a new application. In addition this method have a short simulation time which tolerates its implementation in filtering optimization algorithm.

In future work, filter optimization using this method will be studied. Moreover, part of the studies will be dedicated to simulating the output voltages of the converter in order to have 


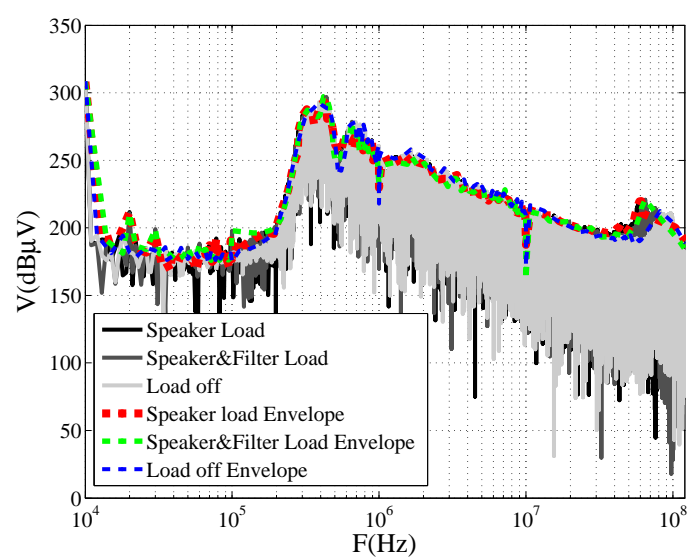

Fig. 17. Class-D amplifier output voltages with different loads.

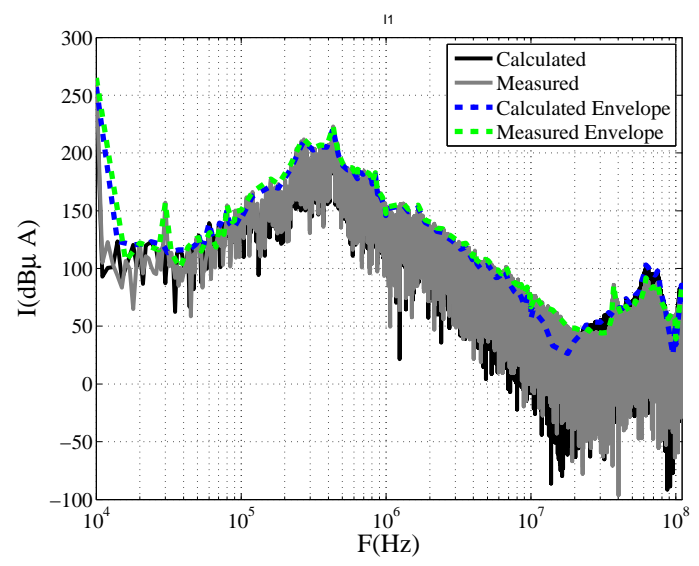

Fig. 18. Calculated current.

a fully predictive method.

\section{APPENDIX A}

PROOF OF EQUATION 8

In order to prove equation 8 , the $k$ and $m$ blocks of sectionII-D1 were re-considered (Fig. 19). In Fig. $19 V_{i n_{k}}$, $V_{\text {out }_{k}}, V_{\text {in }_{m}}, V_{\text {out }_{m}}, I_{\text {in }_{k}}, I_{\text {out }_{k}}, I_{\text {in }_{m}}$ and $I_{\text {out }}$ are vectors with a dimension equal to $\mathrm{N}$. Those vectors are the voltages or the current for the $\mathrm{N}$ ports of $k$ or $m$ blocks.

By definition :

$$
\begin{array}{r}
V_{\text {in }_{k}}=Z_{k 11} I_{\text {in }_{k}}+Z_{k 12} I_{\text {out }_{k}} \\
V_{\text {out }_{k}}=Z_{k 21} I_{\text {in }_{k}}+Z_{k 22} I_{\text {out }_{k}} \\
V_{\text {in }_{m}}=Z_{m 11} I_{\text {in }_{m}}+Z_{m 12} I_{\text {out }_{m}} \\
V_{\text {out }_{m}}=Z_{m 21} I_{\text {in }_{m}}+Z_{m 22} I_{\text {out }_{m}}
\end{array}
$$

Since the block $k$ output is related to the block $m$ input, then :

$$
\begin{gathered}
V_{\text {out }_{k}}=V_{\text {in }_{m}} \\
I_{\text {out }_{k}}=-I_{i n_{m}}
\end{gathered}
$$

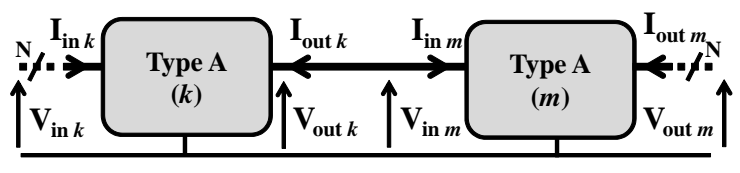

Fig. 19. $\quad k$ and $m$ block association.

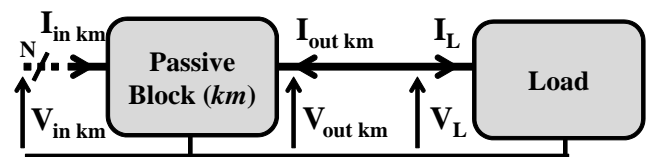

Fig. 20. $\quad \mathrm{km}$ and $L$ block association.

When using (11) and (12) :

$$
\begin{aligned}
V_{\text {out }_{k}} & =-Z_{m 11} I_{\text {out }_{k}}+Z_{m 12} I_{\text {out }_{m}} \\
V_{\text {out }_{m}} & =-Z_{m 21} I_{\text {out }_{k}}+Z_{m 22} I_{\text {out }_{m}}
\end{aligned}
$$

From (11) and (13) :

$$
\begin{aligned}
I_{\text {out }_{k}} & =-\left(Z_{k 22}+Z_{m 11}\right)^{-1} Z_{k 21} I_{i n k_{k}} \\
& +\left(Z_{k 22}+Z_{m 11}\right)^{-1} Z_{m 12} I_{\text {out }_{m}}
\end{aligned}
$$

Finally, when using (14) in (11) and (13)

$$
\begin{gathered}
V_{i n_{k}}=\left[Z_{k 11}-Z_{k 12}\left(Z_{k 22}+Z_{m 11}\right)^{-1} Z_{k 21}\right] I_{\text {in }} \\
+Z_{k 12}\left(Z_{k 22}+Z_{m 11}\right)^{-1} Z_{m 12} I_{\text {out }_{m}} \\
\quad+\left[Z_{m 22}-Z_{m 21}\left(Z_{k 22}+Z_{m 11}\right)^{-1} Z_{m 12}\right] I_{\text {out }_{m}}
\end{gathered}
$$

The resulting block has $V_{i n_{k}}, I_{i n_{k}}$ on the input and $V_{\text {out }_{m}}, I_{\text {out }_{m}}$ on the output. The resulting impedance matrix is the matrix relating $V_{i n_{k}}, V_{o u t_{m}}$ to $I_{i n_{k}}, I_{o u t_{m}}$. Hence, (15) and (16) permit us to deduce equation 8 .

\section{APPENDIX B \\ PROOF OF EQUATION 9}

In order to prove equation 9 , the $k m$ and $L$ blocks of sectionII-D2 were re-considered (Fig. 20). In Fig. 20 $V_{i n_{k m}}, V_{\text {out }_{k m}}, V_{L}, I_{i n_{k m}}, I_{o u t_{k m}}$ and $I_{L}$ are vectors with a dimension equal to $\mathrm{N}$. Those vectors are the voltages or the current for the $\mathrm{N}$ ports of $k m$ or $L$ blocks.

By definition :

$$
\begin{gathered}
V_{\text {in }_{k m}}=Z_{k m 11} I_{\text {in }_{k} m}+Z_{\text {km12 }} I_{\text {out }_{k m}} \\
V_{\text {out }_{k m}}=Z_{k m 21} I_{\text {in }_{k} m}+Z_{k m 22} I_{\text {out }_{k m}} \\
V_{L}=Z_{L} I_{L}
\end{gathered}
$$

Since block $k m$ output is related to block $L$ input, then :

$$
\begin{gathered}
V_{\text {out }_{k m}}=V_{L} \\
I_{\text {out }_{k m}}=-I_{L}
\end{gathered}
$$

When using (18) and (19) :

$$
V_{\text {out }_{k m}}=-Z_{L} I_{\text {out }_{k m}}
$$


Using (20) in (17) :

$$
I_{\text {out }_{k m}}=-\left(Z_{L}+Z_{k m 22}\right)^{-1} Z_{k m 12} I_{i n_{k m}}
$$

Finaly using (21) in (17) :

$V_{i n_{k m}}=\left[Z_{k m 11}-Z_{k m 12}\left(Z_{L}+Z_{k m 22}\right)^{-1} Z_{k m 12}\right] I_{i n_{k m}}$

The resulting block has only $V_{k m L}, I_{k m L}$ on the input. The resulting impedance matrix is the matrix relating $V_{k m L}$ to $I_{k m L}$. Hence, (22) permit us to deduce equation 9.

\section{REFERENCES}

[1] A. Nagari, E. Allier, F. Amiard, V. Binet, and C. Fraisse, "An $8 \Omega$; 2.5W 1\%-THD 104dB(A)-dynamic-range Class-D audio amplifier with an ultra-low EMI system and current sensing for speaker protection," in Solid-State Circuits Conference Digest of Technical Papers (ISSCC), 2012 IEEE International, pp. 92 -94, feb. 2012.

[2] M.-L. Yeh, W.-R. Liou, H.-P. Hsieh, and Y.-J. Lin, "An electromagnetic interference (EMI) reduced high-efficiency switching power amplifier," Power Electronics, IEEE Transactions on, vol. 25, pp. 710 -718, mar. 2010.

[3] M. Xin, C. Zao, Z. Ze-kun, and Z. Bo, "An advanced spread spectrum architecture to improve EMI emissions in Class D amplifier," in Communications, Circuits and Systems, 2009. ICCCAS 2009. International Conference on, pp. $661-665$, jul. 2009.

[4] G. Dousoky, M. Shoyama, and T. Ninomiya, "On factors affecting EMIperformance of conducted-noise-mitigating digital controllers in DCDC converters - An experimental investigation," in Energy Conversion Congress and Exposition (ECCE), 2010 IEEE, pp. $1239-1245$, sept. 2010.

[5] C. F. Edwards, "Efficient low EMI switching output stages and methods," mar. 2007. Patent : US 7190225

[6] T. Lim, H. Muir, S. Finney, and B. Williams, "Adaptive voltage slew control used to limit the magnitude of broadband conducted noise emissions for buck derived dc-dc converters," in Electromagnetic Compatibility (APEMC), 2012 Asia-Pacific Symposium on, pp. 93 -96, may 2012

[7] T. Nakagawa and K. Osada, "Pulse position/width collaborative control for reducing EMI noise and ripple voltage in switching DC-DC converter," in Applied Power Electronics Conference and Exposition (APEC), 2012 Twenty-Seventh Annual IEEE, pp. 1112 -1116, feb. 2012.

[8] R. Kirlin, C. Lascu, and A. Trzynadlowski, "Shaping the noise spectrum in power electronic converters," Industrial Electronics, IEEE Transactions on, vol. 58, pp. $2780-2788$, july 2011.

[9] R. Tallam, R. Kerkman, D. Leggate, and R. Lukaszewski, "CommonMode voltage reduction PWM algorithm for AC drives," Industry Applications, IEEE Transactions on, vol. 46, pp. 1959 -1969, sept.-oct. 2010.

[10] D. Labrousse, B. Revol, and F. Costa, "Common-mode modeling of the association of switching cells: Application to an electric-vehicle-drive system," Power Electronics, IEEE Transactions on, vol. 25, pp. 2852 -2859 , nov. 2010.

[11] C. Jettanasen, F. Costa, and C. Vollaire, "Common-mode emissions measurements and simulation in variable-speed drive systems," Power Electronics, IEEE Transactions on, vol. 24, pp. 2456 -2464, nov. 2009.

[12] F. Costa, C. Vollaire, and R. Meuret, "Modeling of conducted common mode perturbations in variable-speed drive systems," Electromagnetic Compatibility, IEEE Transactions on, vol. 47, pp. 1012 - 1021, nov. 2005.

[13] C. Zheng, C. Chen, and H. Chen, "A simulation platform of switchedmode power supplies for EMI filter design," in Electromagnetic Compatibility (APEMC), 2012 Asia-Pacific Symposium on, pp. 641 -644, may 2012.

[14] J. Meng, X. Zhang, A. Hu, L. Zhang, and W. Ma, "Transfer network models for EMI coupling paths characterization of multiconductor cables," in Electromagnetic Compatibility (APEMC), 2010 Asia-Pacific Symposium on, pp. $301-304$, apr. 2010.

[15] F. Grassi, S. Pignari, and J. Wolf, "Channel characterization and EMC assessment of a PLC system for spacecraft DC differential power buses," Electromagnetic Compatibility, IEEE Transactions on, vol. 53, pp. 664 -675 , aug. 2011.

[16] J.-R. Regue, M. Ribo, D. Duran, D. Badia, and A. Perez, "Common and differential mode characterization of EMI power-line filters from s-parameters measurements," in Electromagnetic Compatibility, 2004. EMC 2004. 2004 InternationalSymposium on, vol. 2, pp. 610 - 615 vol.2, aug. 2004.
[17] J. Hagmann and S. Dickmann, "Determination of mode conversion on differential lines," in Electromagnetic Compatibility - EMC Europe, 2008 International Symposium on, pp. 1 -5, sept. 2008.

[18] M. Reuter, S. Tenbohlen, W. Kohler, and A. Ludwig, "Impedance analysis of automotive high voltage networks for EMC measurements," in EMC Europe 2011 York, pp. 106 -111, sept. 2011.

[19] M. Foissac, J. Schanen, G. Frantz, D. Frey, and C. Vollaire, "System simulation for EMC network analysis," in Applied Power Electronics Conference and Exposition (APEC), 2011 Twenty-Sixth Annual IEEE, pp. $457-462$, mar. 2011.

[20] J. Miyashita, M. Mitsuzawa, T. Karube, K. Yamasawa, and T. Sato, "Measurement of the source impedance of conducted emission using mode separable lisn: Conducted emission of a switching power supply," Electrical Engineering in Japan, vol. 139, no. 2, pp. 72-78, 2002.

[21] V. Tarateeraseth, K. Y. See, F. Canavero, and R. Chang, "Systematic electromagnetic interference filter design based on information from incircuit impedance measurements," Electromagnetic Compatibility, IEEE Transactions on, vol. 52, pp. 588 -598, aug. 2010.

[22] V. Tarateeraseth, K. Y. See, L. B. Wang, and F. Canavero, "Systematic power line emi filter design for smps," in EMC Europe 2011 York, pp. $586-591$, sept. 2011.

[23] Y. Panov and M. Jovanovic, "Practical issues of input/output impedance measurements in switching power supplies and application of measured data to stability analysis," in Applied Power Electronics Conference and Exposition, 2005. APEC 2005. Twentieth Annual IEEE, vol. 2, pp. 1339 -1345 Vol. 2, march 2005.

[24] T. Ibuchi and T. Funaki, "A study on emi noise source modeling with current source for power conversion circuit," in Electromagnetic Compatibility (EMC EUROPE), 2012 International Symposium on, pp. 1 -6 , sept. 2012.

[25] “ADS Advenced Design System." Accessed: 07/19/2012.

[26] Agilent, "Advanced impedance measurement capability of the RF I$\mathrm{V}$ method compared to the network analysis method," Tech. Rep. Application Note 1369-2, Agilent Technologies, Jul. 2001.

[27] Agilent, "Ultra-Low Impedance Measurements Using 2-Port Measurements," Tech. Rep. Application Note 5989-5935EN, Agilent Technologies, Feb. 2007.

[28] F. Lafon, Techniques and methodologies development to take into account EMC constraints in Automotive equipment design. $\mathrm{PhD}$ thesis, INSA de Rennes, 2011.

[29] X. Ye, "De-embedding errors due to inaccurate test fixture characterization," Electromagnetic Compatibility Magazine, IEEE, vol. 1, pp. 75 -78, quarter 2012.

[30] M. Ferber, C. Vollaire, L. Krahenbuhl, J.-L. Coulomb, and J. A. Vasconcelos, "Conducted interferences of power converters with parametric uncertainties in the frequency domain," in Electromagnetic Compatibility (APEMC), 2012 Asia-Pacific Symposium on, pp. 681-684, may 2012.

[31] R. Mrad, F. Morel, G. Pillonnet, C. Vollaire, and D. Labrousse, "Differential passive circuit modelling with pentapole impedance matrices - application to an integrated audio switching amplifier for portable devices," in EMC Europe 2011 York, pp. 304 -309, sept. 2011.

[32] R. Mrad, F. Morel, G. Pillonnet, C. Vollaire, and A. Nagari, "Conducted EMI prediction for integrated Class D audio amplifier," in Electronics, Circuits and Systems (ICECS), 2011 18th IEEE International Conference on, pp. $390-393$, dec. 2011.

[33] M. Berkhout, "Class D audio amplifiers in mobile applications," in Circuits and Systems, 2009. ISCAS 2009. IEEE International Symposium on, pp. $1169-1172$, may 2009.

[34] G. Pillonnet, R. Cellier, N. Abouchi, and M. Chiollaz, "A high performance switching audio amplifier using sliding mode control," in Circuits and Systems and TAISA Conference, 2008. NEWCAS-TAISA 2008. 2008 Joint 6th International IEEE Northeast Workshop on, pp. 305 -309, jun. 2008.

[35] “MAXIM : MAX9700B Evaluation Kit." Accessed: 08/01/2012.

[36] “MEGATRON, NXP Speaker RA11x15x3.5." Accessed: 08/01/2012.

[37] S. Zangui, B. Vincent, K. Berger, R. Perrussel, E. Clavel, C. Vollaire, and O. Chadebec, "Near-field coupling between EMC filter components," in Electromagnetic Field Computation (CEFC), 2010 14th Biennial IEEE Conference on, p. 1, may 2010.

[38] "LeCroy : WaveRunner HRO $64 \mathrm{Zi}$ 12-bit oscilloscopes, $400 \mathrm{MHz}$, website $=$ http://www.lecroy.com/oscilloscope/, note = Accessed: 08/01/2012,."

[39] “Tektronix : current probe, $120 \mathrm{MHz}$, website = http://www1.tek.com/ products/accessories/current.html, note = Accessed: 08/01/2012,." 


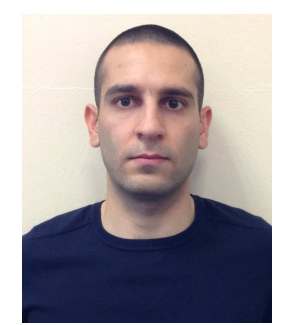

Roberto Mrad was born in Zgharta Lebanon in 1987. He received his master's degree in micro and embedded systems in July 2010 from the Lebanese University. In 2011 he started a PhD with STEricsson (Grenoble-France), the Ampere laboratory (Ecole Centrale de Lyon-France) and the INL laboratory (CPE-Lyon-France). The main topic of his research activities is the conducted EM emissions from switching audio amplifier and power converters.

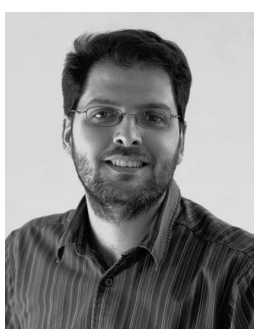

Florent Morel (S'06-M'07) received the Aggregation in electrical engineering from the Ecole Normale Superieure (ENS), Cachan, France, in 2002. Then he received the M.S. and Ph.D. degrees in electrical engineering from the Institut National de Sciences Appliques (INSA), Lyon, France, in 2004 and 2007, respectively. His Ph.D. thesis deals with predictive control, permanent magnet synchronous machines, voltage source inverters and matrix converters.

$\mathrm{He}$ is currently with the laboratory AMPERE and the Ecole Centrale, Lyon, France as an associate professor. His research interests include power electronics and electromagnetic compatibility.

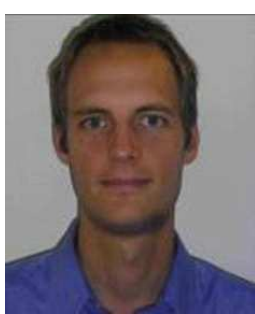

Gael Pillonnet was born in Lyon, France, in 1981. $\mathrm{He}$ received his HE degree in Electrical Engineering from CPE Lyon in 2004 and a $\mathrm{PhD}$ degree from INSA Lyon in 2007. He is now associate Professor at University of Lyon, CPE department. He was visiting researcher at University of California at Berkeley in 2011-12. His research interests include integrated power converter and audio amplifier.

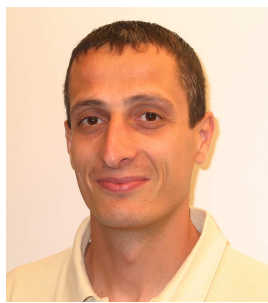

Christian Vollaire was born in 1968. He received the M.S. degree from the University of Saint Jrme, Marseille, France, in 1992 and the Ph.D. degree from the Ecole Centrale de Lyon, Ecully, France, in 1997, both in electrical engineering.

In 1998, he joined the AMPERE Laboratory (UMR CNRS 5005). He is now a Professor at the Ecole Centrale de Lyon. Since then, he has carried out research with AMPERE at the Ecole Centrale de Lyon in the field of the numerical modeling applied to the interaction between electromagnetic field and complex systems. He develops, in particular, specific formulations and numerical methods for the computation of electromagnetic fields in complex structures. One of the fields of applications relates to electromagnetic compatibility.

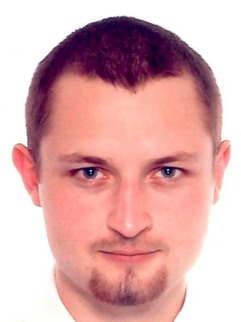

Philippe Lombard was born in France in 1981. He received the M.Sc. degree in microwave engineering from the National Polytechnic School of Grenoble in 2002, and his Ph. D. in Optics and RadioFrequencies from the Joseph Fourrier University of Grenoble-France, in 2007. He held a postdoctoral position, in 2008 at the IEMN Institute, Lille-France. $\mathrm{He}$ is now associate Professor at University of Lyon (INL-UCBL). His current field of interest focuses on micro energy recycling and micro-power converter, electronic modeling and architecture, integrated circuits design in embedded systems.

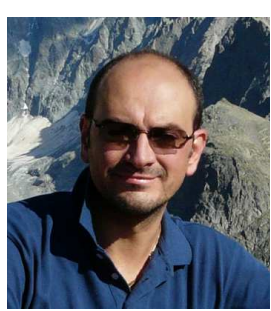

Angelo Nagari Angelo Nagari (IEEE membership: S'92-M'96) was born in Cilavegna, Pavia, Italy, in 1968. He received the degree in electronic engineering (summa cum laude) from the University of Pavia, Italy, in 1993. He has been with STMicroelectronics, Milan, Italy, since 1993, where he was involved as a Design Engineer in the analog and mixed IC development for cellular telecommunications. His main research interests are in the fields of Nyquist and Oversampled converters for System-onChip in Audio, RF and Auxiliary applications. Since February 2008 is IP design manager in ST-Ericsson, Grenoble, France. His main role is to define mixed-signal architecture and partitioning for mobile phones platforms and provides IP design on Audio and Power Management fields. Recently has been appointed "Analog \& System IP" Core Competence responsible for ST-Ericsson analog system development area, having as a main objective to drive all related R \& D development. He's reviewer of several IEEE journals (TCAS, JSSC) and conferences (ISCAS, ESSCIRC) and, within ST-Ericsson, he holds several patents. 